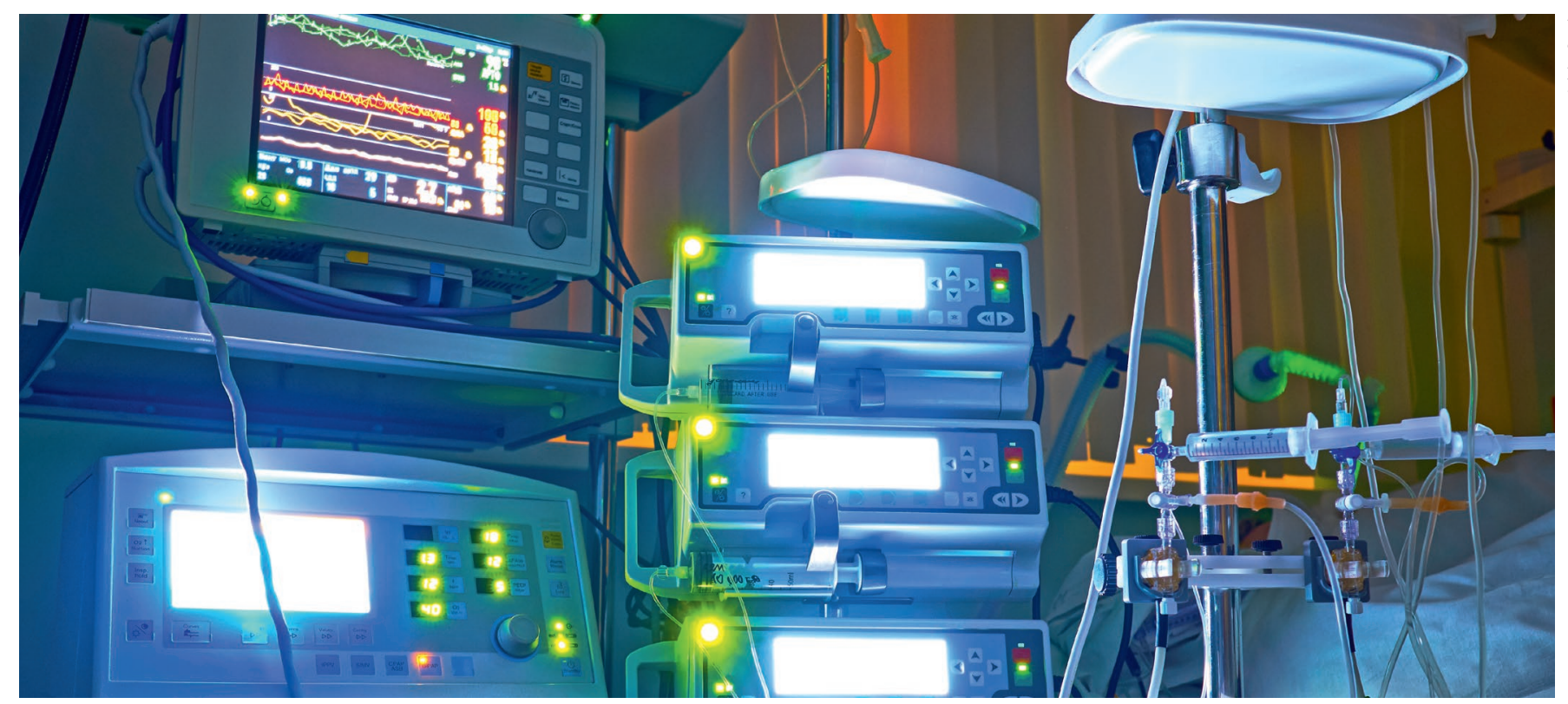

\title{
Die Zukunft der Intensivmedizin
}

Anlässlich des Tages der Intensivmedizin publizierte die ÖGARI ein Positionspapier, wie die Erfahrungen aus der Corona-Pandemie zur weiteren Optimierung der Versorgung genutzt werden sollten.

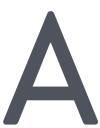
m 20. Juni wurde der „Tag der Intensivmedizin" begangen - ein Anlass, um die Aufgabengebiete und Leistungen dieses wichtigen Spezialgebietes der Medizin aufzuzeigen.

Die Corona-Pandemie hat für eine breite Öffentlichkeit das Fach Anästhesie und Intensivmedizin als Rückgrat der Spitalsversorgung besonders sichtbar gemacht. Zahlreiche Maßnahmen der Pandemiebewältigung wurden mit Blick darauf unternommen, eine Überforderung der Intensivkapazitäten zu vermeiden. Für viele Menschen wurde deutlich und besser verständlich, was die moderne Intensivmedizin leistet, und dass sie nicht auf Gerätemedizin reduzierbar ist, sondern dass der menschliche Faktor eine zentrale Rolle spielt.

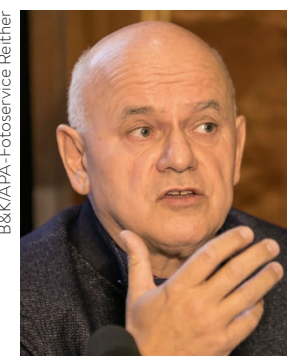

Prim. Univ.-Prof. Dr Walter Hasibeder
„Österreich hat, auch im internationalen Vergleich, generell ein hervorragendes Gesundheitssystem, und es ist insbesondere auch gut aufgestellt, was die intensivmedizinische Versorgung betrifft. Diese Ausstattung und das enorme Engagement aller in unserem Fachgebiet tätigen Gesundheitsberufe - oft an der Grenze der Belastbarkeit - waren Vorteile in der Krisenbewältigung", sagt ÖGARI-Präsident Prim. Univ.-Prof. Dr. Walter Hasibeder, Vorstand der Abteilung für Anästhesie und operative Intensivmedizin am Krankenhaus St. Vinzenz in Zams. Aktuellen Daten zufolge liegt Österreich im OECD-Vergleich mit 28,9 Intensivbetten pro 100.000 Einwohner*innen nach Deutschland und vor den USA an der Spitze, und zum Beispiel deutlich vor Nachbarländern wie der Schweiz (11,8), Ungarn (11,2) oder Italien $(8,6)$. Österreich verfügte Ende Mai 2021 über 2.057 Intensivbetten (Quelle: BMSGPK). Rund 120 Intensivstationen sind in der Versorgung aktiv.

Die Corona-Pandemie hat auch in Österreich das Bewusstsein dafür geschärft, wie wertvoll ausreichende Ressourcen sind, die eine Intensivmedizin auf qualitativ höchstem Niveau gewährleisten. Die Erfahrungen aus der Pandemie und den Spitzenbelastungen der vergangenen Monate sollten nun dafür genutzt werden, heißt es in einem von der ÖGARI entwickelten und publizierten Positionspapier, „die österreichische Intensivmedizin nicht nur bestmöglich für potenzielle künftige Krisensituatio- nen vorzubereiten, sondern generell zukunftssicher zu gestalten".

\section{STÄRKUNG UND AUSBAU}

Es sei wichtig, jetzt sicherzustellen, heißt es in dem Papier, dass die Intensivmedizin nicht nur leistungsfähig bleibt, sondern dass auch, wo immer möglich, an weiteren Optimierungen gearbeitet wird, sodass die Intensivmedizin für künftige demographische und medizinische Entwicklungen gerüstet ist. Wie die Intensivmedizin weiterentwickelt und gestärkt werden kann, müsse Gegenstand eines ,intensiven Austauschs aller interessierten Kräfte" sein. Folgende konkrete Maßnahmen werden in dem Positionspapier angeführt:

- Starke Veränderungen in der Altersstruktur der Patient*innen, die Zunahme der Zahl und Schwere der Vorerkrankungen und die Tatsache, dass erfolgreiche Behandlungen heute auch bei Menschen mit eingeschränkten Leistungsreserven möglich geworden sind, sollten sich in künftigen Anpassungen des Österreichischen Strukturplan Gesundheit (ÖSG) stärker widerspiegeln.

- Es ist wichtig, mit Blick auf mögliche künftige Krisen vorsorglich flexible, abgestufte Strukturen zu schaffen, 
die im Notfall für die Intensivversorgung mobilisierbar sind - auf der Ebene von Betten und Geräten ebenso wie im Hinblick auf die personelle Ausstattung. Im Krisenfall müssen entsprechende Verordnungen für Rechtssicherheit beim Einsatz flexibler Ressourcen sorgen.

- Erforderlich ist eine Nachschärfung bei den Struktur- und Qualitätsmerkmalen für die personelle und technische Ausstattung von Intensivbehandlungseinheiten der verschiedenen Kategorien. Aus Sicht der ÖGARI benötigt ein belastungsstabiles System bundesländerübergreifende Mindeststandards für die personelle Ausstattung des gesamten Anästhesiebereichs mit seinen Operationssälen, Aufwachräumen bzw. Holding Areas.

- Aufwachräume und Holding Areas, in denen im Regelfall die Nachbetreuung von unmittelbar postoperativen Patient*innen erfolgt, können, wie die aktuelle Pandemie gezeigt hat, rasch zu intensivmedizinischen "Noteinrichtungen" umfunktioniert werden. Allerdings scheinen diese Bereiche im aktuellen ÖSG nicht auf; die Finanzierung muss daher durch die Krankenhäuser selbst erfolgen. Diese Räumlichkeiten stellen also eine wichtige intensivmedizinische Reserve dar und benötigen eine gesundheitsplanerische und finanzielle Absicherung.

- Die Herausforderungen der Pandemie haben die Notwendigkeit deutlich gemacht, im Krisenfall zusätzliche Personalreserven für die Intensivversorgung mobilisieren zu können, zum Beispiel ärztliches und Pflegepersonal aus dem Bereich Anästhesie. Hier bewährt sich das österreichische Modell, ärztliches Personal in der Intensivmedizin auf der Basis eines Grundlagenfaches wie Anästhesie oder Innerer Medizin auszubilden, statt im Rahmen einer eigenen Facharztausbildung. Das sichert nicht nur flexible Einsatzmöglichkeiten von qualifiziertem Personal, sondern auch die Attraktivität des Fachs für ausreichenden und gut qualifizierten professionellen Nachwuchs. In ähnlicher Weise sollten aus Sicht der
ÖGARI die pflegerischen Spezialausbildungen in Anästhesie- und Intensivpflege zumindest in Teilbereichen über ein gemeinsames theoretisches und praktisches Curriculum verfügen, damit insbesondere in Krisenzeiten ein flexibler Einsatz dieses hochqualifizierten Personals möglich ist.

- Generell muss die Zukunft der Intensivmedizin immer auch die Zukunft der Intensivpflege mit betrachten, und es müssen alle Potenziale genutzt werden, um eine Tätigkeit in der Intensivpflege attraktiv zu machen.

- Um die Verbleiberate im Beruf zu erhöhen, ist es erforderlich, die Gesunderhaltung des Intensivpersonals aller Berufsgruppen optimal zu unterstützen, z. B. durch Supervision oder Coaching.

- Die Aufmerksamkeit für COVID19-Patient*innen hat für viele deutlich gemacht, dass eine kritische Erkrankung nicht mit der Entlassung aus der Intensivstation endet. Es gilt, den gesamten Patient*innenweg in die Intensivmedizin und nach dem ICU-Aufenthalt noch besser zu strukturieren: Dazu gehört die Vorbereitung von Standards und Leitfäden für strukturierte, diagnoseunabhängige und prognosegestützte Entscheidungen über ICU-Aufnahmen und Therapiezieländerungen ebenso wie eine bestmögliche Begleitung in die Phase der Rekonvaleszenz. Auch die Förderung von Advanced Care Planning ist ein wichtiges Element davon.

- Die erhöhte Sensibilität für die Intensivmedizin in der Öffentlichkeit sollte für weitere Informationsarbeit und Gesundheitsbildung genutzt werden, um noch mehr Verständnis für die intensivmedizinischen Auf- gaben und Abläufe zu schaffen und der Bevölkerung evidenzgesicherte Information weiterzugeben.

- Die Krise hat den potenziellen Nutzen einer erweiterten Intensivdokumentation deutlich gemacht. Es sollten die technischen Voraussetzungen geschaffen werden, um das bestehende Dokumentationssystem in der Intensivmedizin auszubauen (z. B. automatische Übernahme verfügbarer Daten). Auf diesem Weg sollten sich epidemiologische Fragestellungen oder Fragen der intensivmedizinischen Forschung unkomplizierter beantworten und finanzierungsrelevante Aspekte besser abbilden lassen. Die starke Gerätezentrierung der aktuellen Dokumentation und Abgeltung sollte an die Prinzipien der modernen, menschenzentrierten und personalintensiven Intensivmedizin mit weniger Invasivität angepasst werden.

- Eine optimale Nutzung von Informationstechnologien, Big Data und Künstlicher Intelligenz könnte intensivmedizinische personelle Ressourcen schonen und die Versorgung stärken. Smart Monitoring und der Ausbau telemedizinischer Möglichkeiten sind Beispiele dafür. Informationstechnologien sollten auch verstärkt dazu genutzt werden, den Kontakt und die Kommunikation von Intensivpatient*innen mit ihren Anund Zugehörigen aufrechtzuerhalten.

Diese und weitere Fragen sollten in einem intensiven Austausch mit allen interessierten Kräften diskutiert werden, sagt Prof. Hasibeder. „Die ÖGARI steht jedenfalls bereit, an der weiteren Stärkung und dem Ausbau der Intensivmedizin zentral mitzuwirken."

Redaktion

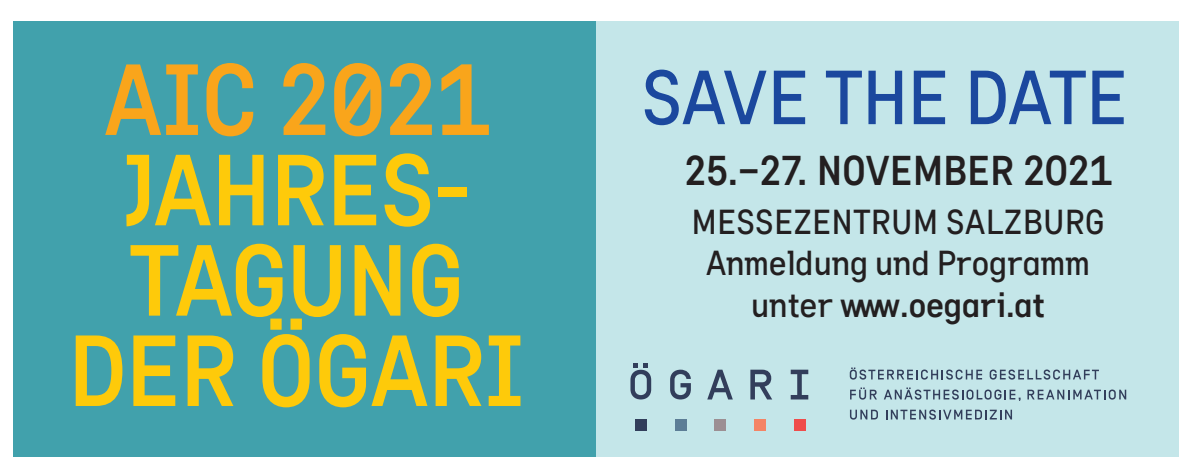

